\title{
COVID-19 e os desafios para a regulação de medicamentos em tempos de pandemia
}

\author{
COVID-19 and the medicines regulation challenges \\ in times of pandemic
}

Vera Lúcia Edais Pepe (https://orcid.org/0000-0003-0606-1544) ${ }^{1}$

Hillegonda Maria Dutilh Novaes (https://orcid.org/0000-0001-9849-0324) ${ }^{2}$

Claudia Garcia Serpa Osorio-de-Castro (https://orcid.org/0000-0003-4875-7216) ${ }^{3}$

\footnotetext{
${ }^{1}$ Departamento de Administração e Planejamento em Saúde, Escola Nacional de Saúde de Saúde Pública Sergio Arouca (ENSP), Fundação Oswaldo Cruz (Fiocruz). R. Leopoldo Bulhões $1480,7^{\circ}$ andar, Manguinhos. 21041-210 Rio de Janeiro RJ Brasil. verapepe01@gmail.com ${ }^{2}$ Faculdade de Medicina, Universidade de São Paulo. São Paulo SP Brasil. ${ }^{3}$ Departamento de Políticas de Medicamentos e Assistência Farmacêutica, ENSP, Fiocruz. Rio de Janeiro RJ Brasil.
}

\begin{abstract}
The SARS-CoV-2 pandemic has brought challenges related to prevention, protection and care. Coping strategies, such as social distancing, individual protection for the population and workers, increase in the number of intensive care beds, provision of human resources and equipment are necessary actions. However, there are yet no specific effective and safe medicines that justify their use. The challenge imposed on the regulatory framework for medicines is aimed at providing timely access to medicines capable of modifying the course of the disease and leading to better treatment outcomes, with health safety. Regulatory agencies must protect the health by assessing the actual benefits and harms of the medicines under these specific conditions. The article discusses the main regulatory challenges and response of regulatory agencies to the demands imposed by the COVID-19 pandemic, especially, drug development strategies and regulatory strategies related to off-label use. Emergency drug use authorization and alternatives for extended/compassionate use are addressed, as well as clinical trials, safety assessment and monitoring of adverse events.
\end{abstract}

Key words COVID-19, Pharmaceutical Preparations, Pandemics, Health Surveillance
Resumo A pandemia de SARS-CoV-2 trouxe desafios relacionados à prevenção, proteção e cuidado. Estratégias de enfrentamento, como distanciamento social, medidas de proteção individual da população e trabalhadores, ampliação dos leitos de terapia intensiva, disponibilização de recursos humanos e equipamentos são ações necessárias. Não há, ainda, medicamentos específicos com eficácia e segurança que justifiquem sua utilização. O desafio imposto ao marco regulatório de medicamentos volta-se para o acesso tempestivo a medicamentos capazes de modificar o curso da doença e conduzir a melhores desfechos no tratamento, com segurança sanitária. Cabe às agências reguladoras a proteção da saúde com a atribuição de avaliar os reais benefícios e malefícios dos medicamentos nestas condições especificas. $O$ artigo apresenta as ações das agências reguladoras $e$ discute os desafios na implementação da política regulatória de medicamentos frente às exigências impostas pela pandemia de COVID-19. São abordadas, especialmente, estratégias de desenvolvimento de fármacos e estratégias regulatórias sobre a indicação de uso off label, do uso emergencial de medicamentos e das alternativas de uso extendido/compassivo, bem como da realização de ensaios clínicos e da avaliação da segurança e monitoramento de eventos adversos.

Palavras-chave COVID-19, Preparações Farmacêuticas, Pandemias, Vigilância Sanitária 


\section{Introdução}

Um novo tipo de coronavírus, SARS-CoV-2, foi detectado na China, em 2019, responsável pela COVID-19. Em janeiro de 2020 foi declarada, pela Organização Mundial da Saúde (OMS), Emergência de Saúde Pública de Importância Internacional (ESPII), e em março de 2020, pande$\mathrm{mia}^{1}$. Até 14 de maio de 2021 foram confirmados 160.813.869 casos da doença e 3.339.002 óbitos no mundo. No Brasil, 15.359.397 casos e 428.034 óbitos ${ }^{2}$.

São assintomáticos cerca de $80 \%$ dos afetados, e metade dos sintomáticos evolui para forma grave, necessitando de internação ou tratamento intensivo ${ }^{3}$. Os sistemas de saúde enfrentaram e ainda enfrentam grandes dificuldades no rastreamento e atenção aos casos leves e principalmente aos casos hospitalizados, com recursos estruturais e humanos muitas vezes aquém das necessidades e utilização de protocolos de cuidado efêmeros. Estratégias, como distanciamento social, proteção individual da população e de trabalhadores, fluxos de assistência, leitos de terapia intensiva, recursos humanos especializados e equipamentos médicos continuam desafios crescentes conforme a epidemia avança por ondas, com intensidade e distribuição temporal distintas nos diferentes países.

Algumas características do vírus já são conhecidas, restando ainda incertezas sobre a história natural da doença, suas formas em cada indivíduo e o resultado da imunização $0^{5}$. As principais medidas farmacológicas, como corticoides e anticoagulantes, ainda se constituem nos tratamentos adjuvantes que vêm demonstrando eficácia em certas fases da doença ${ }^{6}$. Outras, embora autorizadas por entidades médicas no âmbito da relação de prestação de serviços, entre profissionais e pacientes ${ }^{7}$, caracterizam-se por empirismo e uso off-label ${ }^{4,8}$. Tal emprego é incentivado por forte pressão para disseminação de uso de fármacos já conhecidos para outras indicações (reposicionados), havendo preocupação com a segurança, já que podem estar associados a eventos adversos graves, nas doses de algumas recomendações offlabel ${ }^{6,8}$.

Cabe às agências reguladoras a proteção da saúde na perspectiva coletiva e, no que tange aos medicamentos, têm importante atribuição de avaliar reais benefícios e malefícios do tratamento. Procuram propiciar o acesso tempestivo aos medicamentos necessários, e ao mesmo tempo evitam que medicamentos com possíveis eventos adversos graves sejam utilizados de ma- neira indevida, para não submeter pacientes a riscos à saúde. A avaliação do benefício e do risco dos novos medicamentos e novas indicações terapêuticas permanece necessária mesmo por muitos anos após o registro, de forma a apoiar a inovação e ao mesmo tempo preservar a Saúde Pública ${ }^{9,10}$. As agências reguladoras, assim, foram muitas vezes consideradas burocráticas e com exigências de evidências clínicas muito bem estabelecidas, levando a respostas mais demoradas, que estariam prejudicando a incorporação de novas tecnologias potencialmente efetivas nos sistemas de saúde.

O marco regulatório dos medicamentos sofreu mudança importante, no mundo, após 2012. O desenvolvimento de medicamentos biológicos, especialmente para doenças raras e graves, tem levado à flexibilização das exigências regulatórias $^{11}$. Torna-se cada vez mais necessário gerar informações de segurança após a comercialização para corroborar o acerto da decisão de registro precoce $^{12}$.

As emergências sanitárias, em especial as relacionadas às doenças infecciosas, trazem novos e importantes desafios para as agências reguladoras. Nestes contextos, o acesso a vacinas e medicamentos, que possam responder à doença, é urgente, sendo escasso o tempo entre a regulação e o uso. Nestes momentos, o sistema de saúde encontra-se no limite de suas capacidades, a comunidade temerosa e os próprios profissionais em risco. É com base nisso que se torna importante discutir criticamente, com base em exemplos selecionados, as dificuldades enfrentadas pelas agências reguladoras.

Busca-se aqui apresentar as providências tomadas pelas agências reguladoras e discutir os desafios na implementação da política regulatória de medicamentos frente às exigências impostas pela pandemia de COVID-19, especialmente no Brasil. Toma-se por base exemplos selecionados na literatura, para organizar e dar sentido às evidências, tendo em vista a profusão, a grande variabilidade metodológica, as condições adversas para a realização dos estudos clínicos, bem como a pressão sobre os serviços de saúde e as dificuldades na organização da atenção clínica.

\section{Acesso a medicamentos e regulação: formas tradicionais e aceleradas de registro, e uso sem registro}

O desenvolvimento dos medicamentos é longo, caro e repleto de incertezas ${ }^{13}$. As pesquisas clínicas são necessárias para estabelecer as evidên- 
cias de eficácia e segurança. Nos estudos de fase IV, após o registro, são confirmados (ou não) os benefícios clínicos, estabelecendo, de forma mais adequada, o valor terapêutico do medicamento. O benefício e o risco dos novos medicamentos ou novas indicações terapêuticas só se tornam evidentes quando utilizados por muitos indivíduos e por longo período ${ }^{9,10}$.

As agências reguladoras, como a americana Food and Drug Administration (FDA), a Europeia (EMA) e a Agência Nacional de Vigilância Sanitária (Anvisa), já ofereciam antes da pandemia formas de antecipar o acesso, acelerando o tempo até o registro ${ }^{14}$. O FDA permite: (i) utilizar desfechos substitutos (accelerated approval); (ii) reduzir tempo de análise no caso de doenças graves, ou quando não há alternativa disponível (priority review); (iii) maior interação entre o FDA e a empresa permitindo pedido de registro a qualquer momento (fast track); (iv) uso médico especial, quando o medicamento é considerado revolucionário, proposto para uma condição potencialmente fatal ou com evidências preliminares de benefício, frente às alternativas terapêuticas existentes (breakthrough therapy $)^{15}$.

A European Medicines Agency (EMA) tem modalidades similares. Em março de 2016, instituiu o programa PRIME (PRIority MEdicines), de forma a dar suporte ao desenvolvimento de medicamentos para o tratamento de necessidades para as quais não há qualquer alternativa (unmet need), otimizando planos de desenvolvimento, coleta de dados mais robustos e submissão de pedido de registro com maior qualidade ${ }^{16}$.

A Anvisa prevê a priorização de registro e a anuência prévia de pesquisa clínica sendo critérios para a priorização, dentre outros, os medicamentos utilizados para doenças raras, negligenciadas, emergentes ou reemergentes ${ }^{17}$.

Formas alternativas de acesso a medicamentos, antes do registro sanitário, têm sido utilizadas. Em doenças raras e câncer, onde o tempo é crucial, a possibilidade de acesso a medicamentos, em estudos de Fase III ou II, se dá por meio do acesso expandido (ou uso expandido - a nomenclatura varia conforme a agência reguladora), seja individual ou em grupo ${ }^{15,18}$. A Lei "Right to Try", desde 2018, permite ao FDA oferecer o acesso de pacientes graves a medicamentos cujo estudo de Fase I já tenha finalizado ${ }^{15}$.

No Brasil, o acesso expandido, para grupos de pacientes, se dá durante ou após estudos fase III. Existe também o uso compassivo, para indivíduos com doenças debilitantes graves e/ou que ameacem a vida, para as quais não existe alterna- tiva terapêutica no país. Neste caso, o uso pode se dar nas fases anteriores da pesquisa clínica ${ }^{19}$.

\section{Quanto tempo o tempo tem? O registro de medicamentos em tempos de pandemia}

Nas condições habituais, o preceito ético da pesquisa com tecnologias em saúde é de que existe um tempo para a investigação, a ser respeitado. O tempo deve ser aquele necessário à consecução da pesquisa e ao amadurecimento da análise dos resultados, evitando potenciais danos aos pacientes $^{20}$.

A $\mathrm{OMS}^{21}$ considera ser obrigação moral a produção mais rápida possível de conhecimento para a resposta a epidemias, com pesquisas clínicas conduzidas de forma ética. Na epidemia de Ebola, foram realizados ensaios clínicos com tempo reduzido, a despeito dos obstáculos logísticos frente à disseminação da epidemia em condições sanitárias, econômicas e sociais muito adversas $^{22}$.

Um argumento que nasce a partir da epidemia de Ebola, e que trata da questão do uso de medicamentos em emergências, é a percepção de que o paciente é o centro da questão: a pesquisa, que indicaria eficácia e segurança das intervenções, estaria dependente das necessidades prementes do paciente. Ou seja, uma intervenção não comprovada poderia ser utilizada em emergências, dada a necessidade imperiosa de salvar vidas $^{23}$. Esse uso é aproximado ao dos programas de acesso expandido/uso compassivo, onde deve haver monitoramento estreito dos resultados.

O debate em torno da priorização do uso de medicamentos no bojo de ensaio clínico durante uma epidemia, tem sido aperfeiçoado ${ }^{22,24,25}$. Baden et al. $^{24}$ reconhecem a dificuldade de se conduzir ensaios clínicos randomizados durante emergências. É importante distinguir cuidado clínico e pesquisa. O sujeito da pesquisa deve ser informado que o estudo contempla o potencial benefício para outras pessoas no futuro e não necessariamente para seu cuidado clínico individual. Por fim, as informações devem ser rapidamente difundidas e o acesso equitativo aos benefícios da pesquisa deve ser garantido ${ }^{21,25}$.

Organizações internacionais indicam a necessidade de, nos períodos entre as epidemias, desenvolver um marco referencial, de forma a propiciar uma pronta e efetiva resposta a um próximo evento ${ }^{24}$. A OMS ${ }^{21}$ recomenda que a metodologia do estudo deve ser apropriada, evitando expor o sujeito de pesquisa a riscos. Dodd et al. ${ }^{26}$, em metanálise de ensaios realiza- 
dos durante a epidemia de Ebola, identificaram considerável heterogeneidade entre os estudos. Os grupos controles eram muito diferentes, sugerindo que estudos não randomizados não são confiáveis como referência válida para evidência de eficácia. Reforça-se, portanto, a recomendação de que o acesso a medicamentos sem registro ou com nova indicação de uso durante epidemias, seja preconizada prioritariamente no âmbito da pesquisa clínica ${ }^{27}$.

A Autorização de Uso Emergencial (AUE) tem sido, preponderantemente, usada na epidemia como forma alternativa de acesso a tecnologias, ou a indicações não registradas. A EMA, desde 2009, quando da epidemia de H1N1, instituiu o Emergency Authorization Procedure. Nos Estados Unidos, a AUE, segundo o FDA, facilita a disponibilidade e uso de tecnologias necessárias ao enfrentamento de emergências sanitárias e auxilia na proteção da Saúde Pública contra ameaças químicas, biológicas, radioativas e nucleares $^{8}$. A OMS propõe alguns critérios para acessar tratamento por meio do uso emergencial: inexistência de tratamento efetivo; impossibilidade de iniciar estudo clinico imediatamente; dados preliminares disponíveis que suportem eficácia e segurança da intervenção; aprovação das autoridades do comitê de ética locais; recursos disponíveis para garantir que o risco do uso seja minimizado; consentimento informado do paciente; monitoramento do uso com resultados rapidamente compartilhados com a comunidade cientifica ${ }^{21}$.

\section{A regulação de medicamentos na COVID-19: o tempo de resposta à pandemia e as evidências científicas ou "fáticas"}

A evolução do conhecimento sobre a história natural da doença evidenciou fases distintas - viremia, fase inflamatória, estado de hipercoagulação e insuficiência respiratória e, em caso de agravamento, de hiper inflamação e falência re$\mathrm{nal}^{5} \mathrm{e}$ o uso de medicamento reflete essa evolução. Além dos antivirais surgiram como relevantes os medicamentos de suporte, como corticoides, heparinas, anestésicos, vasopressores e antibióticos.

Três abordagens têm sido utilizadas para a descoberta de medicamentos para as epidemias de coronavírus (i) uso de antivirais de amplo espectro, já existentes; (ii) reposicionamento de fármacos a partir de bibliotecas químicas de compostos sintetizados, onde se rastreiam moléculas com possíveis efeitos terapêuticos; (iii) desenvolvimento de medicamentos baseados na informação genômica e nas características do SARS-CoV-2, que segue os tramites usuais de desenvolvimento de um medicamento, podendo levar muitos anos e correndo os riscos inerentes ao processo ${ }^{28}$.

No decorrer da epidemia têm sido realizados muitos estudos em inúmeros países. Em cada região as características da pandemia foram diferentes, seja nos subgrupos populacionais mais acometidos, seja no progresso temporal, seja nas formas mais graves da doença. $E$ os ensaios refletiram essas especificidades ${ }^{28}$.

$\mathrm{Na}$ ausência de tratamento antiviral específico, alguns fármacos com atuação antiviral demonstrada em outras condições clínicas, foram incluídos em estudos clínicos e observacionais. A maior parte já foi introduzida no mercado para outras indicações, razão pela qual são denominados 'reposicionados': cloroquina/hidroxicloroquina, azitromicina, nitazoxanida, ivermectina, lopinavir-ritonavir, olsetamivir, darunavir, rendesivir.

A International Coalition of Medicines Regulatory Authorities (ICMRA), reunindo 28 representantes de agências reguladoras, tem realizado encontros regulares para discutir a atuação regulatória face à COVID-1929. Uma preocupação é o número de ensaios de baixo poder e de estudos observacionais, competindo por recursos e pacientes elegíveis, sem gerar evidências robustas. Rome e Avorn ${ }^{30}$ destacam a importância das agências reguladoras avaliarem rapidamente os resultados de ensaios clínicos, face à realização de estudos com metodologia inadequada e o largo uso de medicamentos sem eficácia ou segurança estabelecidas. Assim evitar-se-ia o legado de desconfiança sobre o processo de avaliação de medicamentos em situações de pandemia ${ }^{20}$. A ICMRA reforça a necessidade de ensaios multicêntricos, com protocolos centralizados (master protocols) e múltiplos braços, inclusive com braço controle ${ }^{31}$. Um exemplo é o ensaio SOLIDARITY, da OMS, que envolve pacientes hospitalizados com participação de mais de 100 países $^{32}$.

A multiplicidade de estudos incapazes de gerar robustas evidências, não contribui para a tomada de decisões das agências reguladoras e tampouco auxilia a decisão clínica nos serviços de saúde, submetidos a alta pressão ${ }^{33}$.

O FDA criou o Programa de Aceleração de Tratamento contra a COVID-19 visando facilitar o desenvolvimento e acesso a potenciais tratamentos para a doença, com estratégias para garantir a boa prática clínica, bem como minimizar o risco. Como é previsto que em situações 
de emergência o protocolo do estudo possa ser modificado, eventuais mudanças devem ser documentadas e informadas aos participantes. As informações sobre eficácia e segurança precisam ser avaliadas, ainda que por estratégias alternativas, quando houver dificuldades de seguir o protocolo $^{34}$. O FDA instituiu um Sistema Sentinela para a COVID-19, de forma a monitorar o curso da doença, a utilização de medicamentos e o impacto dos tratamentos, utilizando, inclusive, dados de mundo real ${ }^{35}$.

A European Medicines Agency (EMA) organizou duas forças tarefas para dar respostas à pandemia: A COVID-19 EMA pandemic Task Force objetiva auxiliar na rápida tomada de decisão e coordenar a ação regulatória relacionada ao desenvolvimento, registro e monitoramento da segurança de tratamentos e vacinas ${ }^{36}$. A COVID-19 Task Force busca responder aos desafios para a atuação cientifica e regulatória, incluindo o alinhamento com parceiros europeus e internacionais. Entre as medidas tomadas pelos task forces, a EMA previu uma série de procedimentos de fast-track para vacinas e medicamentos. Há previsão do procedimento acelerado de registro para as tecnologias de interesse para a Saúde Pública, bem como uso compassivo das tecnologias que ainda não possuem registro na Comunidade Europeia $^{36,37}$. Prevê, também, a utilização de medicamentos já registrados, que estejam submetidos a ensaios clínicos.

\section{O rápido "turn over" de evidências e as agências reguladoras}

A cloroquina e a hidroxicloroquina foram globalmente testadas em diferentes condições. O uso profilático, após exposição, não demonstrou proteção maior que o placebo ${ }^{6,32,38}$. A OMS, frente à falta de evidências de eficácia, advindas de diversos ensaios com resultados preliminares publicados, retirou cloroquina e hidroxicloroquina do ensaio Solidarity ${ }^{32}$. A seguir, Mehra et al. ${ }^{39}$, se retrataram pelo estudo que dava suporte ao uso destas substâncias, por terem empregado dados de utilização hospitalar não validados. A OMS após a publicação de resultados dos ensaios Recovery ${ }^{40}$, retirou os fármacos do ensaio Solidarity. Diversos estudos realizados com estes medicamentos apresentaram resultados que não sustentam sua eficácia e ainda mostram eventos adversos $6,32,38,41$.

Ambos os fármacos, cloroquina e hidroxicloroquina, foram aprovados para Autorização de Uso Emergencial no FDA. Algumas condições seriam necessárias para a AUE, dentre elas a de que o sistema de saúde mantivesse a informação sobre a distribuição do medicamento e apresentasse condições de monitorar e comunicar os eventos adversos e erros de medicação ${ }^{42}$. Em 15 de junho, o FDA revogou a Autorização de Uso Emergencial, tendo como justificativa três grandes ensaios que não encontraram ganhos em sobrevida ou benefícios clínicos ${ }^{43}$.

A EMA, por outro lado, não aprovou o uso desses medicamentos, ficando a decisão no âmbito de estudos clínicos ou em protocolos para cada país da Comunidade Europeia ${ }^{44}$. Além disso, alertou os profissionais de saúde que monitorassem os eventos adversos e os reportassem às autoridades sanitárias de seus países ${ }^{45}$.

No FDA, o rendesivir obteve autorização para investigação de novo medicamento e havia sido aprovado para o uso expandido. O estudo Adaptive COVID-19 Treatment Trial (ACTT), multicêntrico e avaliando o tratamento em pacientes adultos hospitalizados, em cerca de 60 locais do mundo, mostrou, em resultados preliminares, que o uso compassivo de rendesivir resultou um menor tempo de internação ${ }^{46}$. O rendesivir obteve, em maio, a AUE para o tratamento de adultos e crianças hospitalizados, com doença grave. Além do rendesivir, em fins de 2020 e 2021, o FDA concedeu AUE para: baracitinibe, casirivimabe e imdevimabe, bamlanivimabe e etesevi$\mathrm{mabe}^{47}$.

A EMA permitiu o uso compassivo do rendesivir para COVID-19 em pacientes hospitalizados, maiores de 12 anos, portadores de síndrome respiratória aguda grave ${ }^{48}$, e em julho de 2020 concedeu registro condicional, com validade de um ano ${ }^{49}$.

Assim como no resto do mundo, a pandemia do coronavírus, desde sua decretação como Emergência de Saúde Pública no Brasil ${ }^{50}$, vem gerando grande quantidade de informação e de pesquisas clínicas. À medida que efeitos possíveis da hidroxocloroquina e da cloroquina vieram à tona, houve uma corrida da população ao varejo para estocar esses medicamentos, prejudicando pacientes com lúpus, usuários de hidroxicloroquina. A Anvisa agiu rapidamente, inserindo ambos os fármacos em lista de medicamentos sob controle de dispensação ${ }^{51}$. A primeira preocupação da Agência foi em relação ao desabastecimento, decorrente da compra por indicação de uso off label, seja sob prescrição médica ou por automedicação, tendo importantes personagens do país claramente favoráveis à sua utilização. Ivermectina, nitazoxanida, interferon e outros 
fármacos com potencial ação antiviral foram a seguir inseridas na RDC 405/2020, na lista de medicamentos sob prescrição especial ${ }^{52}$. A Anvisa não admitiu a eficácia de hidroxicloroquina, cloroquina ou ivermectina no tratamento da COVID-19 $9^{53,54}$.

A Anvisa também emitiu normas extraordinárias no sentido de agilizar o registro de testes diagnósticos (in vitro), medicamentos e produtos biológicos para prevenção e tratamento da COVID-19. O Brasil admitiu o uso da cloroquina e da hidroxicloroquina nos serviços, a critério médico, secundadas por nota informativa do MS ${ }^{55,56}$, diferentemente do FDA e contrariamente à OMS, fora do ambiente dos ensaios clínicos ou AUE, o que configura uso não racional. A utilização de medicamentos para COVID-19 tem se dado, até o momento, em regime off label, sem protocolos de uso emergencial. Ainda que tenha se preocupado em não chancelar o uso de medicamentos sem evidências robustas de eficácia e segurança, a Anvisa não empregou estratégias de monitoramento de eventos adversos.

Em 2021, a Anvisa concedeu registro condicional ao rendesivir para o tratamento hospitalar de COVID-19 e a AUE para a combinação em doses fixas dos anticorpos monoclonais casirivimabe e imdevimabe, em administração intravenosa, para o tratamento de pacientes ambulatoriais com COVID-19, sem necessidade de suplementação de oxigênio e com risco de progressão para a forma grave da doençç ${ }^{57,58}$. Embora tenham sido registrados, o uso destes medicamentos não foi recomendado pela Comissão de Incorporação de Tecnologias (Conitec), do Ministério da Saúde, para o tratamento de pacientes hospitalizados por COVID-1959. Até o momento não há diretriz da Conitec para o tratamento ambulatorial ou preventivo.

O evoluir da pandemia de COVID-19 tem trazido ensinamentos e desafios. Thomson e Nachlis ${ }^{60}$ propõem estratégias a partir do ocorrido no FDA com a cloroquina/hidroxicloroquina, dentre elas: análise de autorização emergencial/ registro por Comitê Consultivo onde estejam diferentes atores; transparência no processo com participação da população; priorizar o estabelecimento de um robusto monitoramento pós-comercialização que inclua estudos de fase 4 rigoroso e amplo; considerar um Programa Nacional de Compensação de Danos por Vacinas; incrementar a comunicação para a população; envolver especialistas e a comunidade médica; rever e comunicar ao público informações sobre os eventos adversos; estabelecer diferentes padrões para a concessão de AUE: mais rígidos para medicamentos e vacinas e mais flexíveis para testes diagnósticos e produtos que serão utilizados para populações especificas; estabelecer e comunicar as evidências que informarão a concessão e retirada de EUA.

Um dos desafios diz respeito às dificuldades de seguir as diretrizes para a Autorização de Uso Emergencial, tais como a aplicação do Termo de Consentimento Informado, a aprovação por Comitê de Ética Nacional e a geração de informação com o uso da tecnologia ${ }^{61,62}$.

\section{Considerações finais}

A epidemia trouxe consigo a necessidade de se conhecer a história natural da doença, suas principais características, e, também, buscar tratamentos específicos e adequados para cada fase da doença. Fármacos e medicamentos candidatos à efetividade frente à COVID-19 se sucedem, cada qual com suas particularidades: medicamentos já utilizados em outras doenças - em uso emergencial ou em outras formas de uso off label -, e novos medicamentos, na modalidade de acesso expandido/uso compassivo.

A forma alternativa de regular o uso de intervenções em emergências, e especialmente o uso "emergencial" é um encontro possível entre a premência da epidemia e a necessidade de chancela, vigilância ou escrutínio por parte da autoridade regulatória. O FDA e a EMA utilizam essa estratégia, com pressupostos muito bem definidos, monitoramento contínuo e notificação de eventos adversos. Em todo mundo, a tomada de decisão quanto ao uso dos medicamentos tem se mostrado claudicante e, por vezes, responsiva às pressões externas e internas aos países, influenciada pela gangorra científica que envolve a realização intensa de estudos de diversos tipos, de qualidade muitas vezes questionável, gerando resultados por vezes conflitantes ou não conclusivos.

Além da pressão, nas agências reguladoras, pelo registro em situação acelerada, há também pressões sobre gestores, profissionais de saúde e mesmo a população, sob as mais diversas estratégias, para a utilização de novos medicamentos e de uso em indicações não registradas. As grandes empresas internacionais, no Brasil, têm recorrido, também, ao sistema legislativo para garantir seus interesses, mesmo quando há recomendação técnica contrária da agência reguladora. Este aspecto é de suma importância, uma vez que na pós pan- 
demia esses atores se sentirão fortalecidos, eventualmente, para pressionar, cada vez mais, pela desregulamentação e, portanto, por enfraquecer o princípio constitucional da proteção da saúde.

A experiência com as demais epidemias e com a COVID-19, tem preconizado a importância de se utilizar os medicamentos sob pesquisa clínica, colaborativa, multicêntrica, com pergunta precisa e desenho de estudo bem delineado, visando produzir, precocemente, evidências mais robustas. Tais medidas contribuem, igualmente, para o estabelecimento de um marco regulatório mais estável e embasado em evidências no contexto da pandemia.

\section{Colaboradores}

VLE Pepe, HMD Novaes e CGS Osorio-de-Castro contribuíram igualmente na formulação, desenvolvimento, análise, redação e revisão final do texto. 


\section{Referências}

1. World Health Organization (WHO). WHO Director-General's opening remarks at the media briefing on COVID-19 [Internet]. WHO; 2020 [acessado 2020 abr 26]. Disponível em: https://www.who.int/dg/ speeches/detail/who-director-general-s-opening-remarks-at-the-media-briefing-on-covid-19---11-march-2020.

2. World Health Organization (WHO). WHO Coronavirus (COVID-19) Dashboard [Internet]. WHO; 2021. [acessado 2021 maio 14]. Disponível em: https://covid19.who.int/.

3. Castro MC, Carvalho LR, Chin T, Kahn R, França GVA, Macário EM, Oliveira WK. Demand for hospitalization services for COVID-19 patients in Brazil. medRxiv 2020; [preprint].

4. Kalil AC. Treating COVID-19-Off-Label Drug Use, Compassionate Use, and 146 Randomized Clinical Trials During Pandemics. JAMA 2020; 323(19):18971898.

5. Wadman M, Couzin-Frankel J, Kaiser J, Matacic C. How does coronavirus kill? Clinicians trace a ferocious rampage through the body, from brain to toes. Sciencemag; 2020 [acessado 2020 set 30]. Disponível em: https://www.sciencemag.org/news/2020/04/how-does-coronavirus-kill-clinicians-trace-ferocious-rampage-through-body-brain-toes.

6. Siemieniuk RAC, Bartoszko JJ, Ge L, Zeraatkar D, Izcovich A, Kum E, Pardo-Hernandez H, Qasim A, Martinez JPD, Rochwerg B, Lamontagne F, Han MA, Liu Q, Agarwal A, Agoritsas T, Chu DK, Couban R, Cusano E, Darzi A, Devji T, Fang B, Fang C, Flottorp SA, Foroutan F, Ghadimi M, Heels-Ansdell D, Honarmand K, Hou L, Hou X, Ibrahim Q, Khamis A, Lam B, Loeb M, Marcucci M, McLeod SL, Motaghi S, Murthy S, Mustafa RA, Neary JD, Rada G, Riaz IB, Sadeghi$\operatorname{rad}$ B, Sekercioglu N, Sheng L, Sreekanta A, Switzer C, Tendal B, Thabane L, Tomlinson G, Turner T, Vandvik PO, Vernooij RW, Viteri-García A, Wang Y, Yao L, Ye Z, Guyatt GH, Brignardello-Petersen R. Drug treatments for covid-19 living systematic review and metanalysis. BMJ 2020;370:m2980.

7. Conselho Federal de Medicina (CFM). Parecer CFM no 4/2020. Considerar o uso da cloroquina e hidroxicloroquina, em condições excepcionais, para o tratamento da COVID-19 [Internet] 2020 [acessado 2020 abr 16]. Disponível em: https://sistemas.cfm.org.br/normas/ visualizar/pareceres/BR/2020/4.

8. Food and Drug Administration (FDA). Emergency Use Authorization (EUA) information, and list of all current EUAs. 2020 [acessado 2020 out 29]. Disponível em: https://www.fda.gov/emergency-preparedness -and-response/mcm-legal-regulatory-and-policy-framework/emergency-use-authorization.

9. World Health Organization (WHO). Access to new medicines in Europe: technical review of policy initiatives and opportunities for collaboration and research [Internet]. 2015 [acessado 2020 abr 27]. Disponível em: http://apps.who.int/medicinedocs/documents/ s21793en/s21793en.pdf.

10. Wied CCG, Leufkens HGM. From molecule to market access: Drug regulatory science as an upcoming discipline. Eur J Pharmacol 2013; 719:9-15.
11. Osorio-de-Castro CGS, Caetano R, Pepe VLE. The 21st Century Cures Act: can the regulatory framework survive the "cures"? Cad Saude Publica 2015; 31(9):1807-1810.

12. World Health Organization (WHO). Pharmacovigilance indicators: a practical manual for the assessment of pharmacovigilance systems [Internet]. 2015 [acessado 2020 abr 27]. Disponível em: http://www.who.int/ iris/handle/10665/186642.

13. Mohs RC, Greig NH. Drug discovery and development: Role of basic biological research. Alzheimers Dement 2017; 3(4):651-657.

14. Darrow JJ, Avorn J, Kesselheim AS. FDA approval and regulation of pharmaceuticals, 1983-2018. JAMA 2020; 323:164-176.

15. Speers MA. Providing Patients with Critical or Life-Threatening Illnesses Access to Experimental Drug Therapy: A Guide to Clinical Trials and the US FDA Expanded Access Program. Pharmaceut Med 2019; 33(2):89-98.

16. Detela G, Lodge A. EU Regulatory Pathways for ATMPs: Standard, Accelerated and Adaptive Pathways to Marketing Authorisation. Mol Ther Methods Clin Dev 2019; 13:205-232.

17. Agência Nacional de Vigilância Sanitária (Anvisa). RDC 204, de 27 de dezembro de 2017. Dispõe sobre o enquadramento na categoria prioritária, de petições de registro, pós-registro e anuência prévia em pesquisa clínica de medicamentos. Diário Oficial da União 2017; 28 dez.

18. Van Norman GA. Drugs and Devices Comparison of European and U.S. Approval Processes. JACC Basic Transl Sci 2016; 1(5):399-412.

19. Agência Nacional de Vigilância Sanitária (Anvisa). RDC 38, de 12 de agosto de 2013. Aprova o regulamento para os programas de acesso expandido, uso compassivo e fornecimento de medicamento pós-estudo. Brasilia. 2013. Diário Oficial da União 2013; 12 ago.

20. London AJ, Kimmelman J. Against pandemic research Exceptionalism, Science Editorial. Science 2020; 368 (6490):476-477.

21. World Health Organization (WHO). Ethics in epidemics emergencies and disasters training manual [Internet]. 2015 [acessado 2020 out 29]. Disponível em: https://apps.who.int/iris/bitstream/handle /10665/196326/9789241549349_eng.pdf;jsessionid=DDB52CC34CB34AAE2F64AF56ED40101B?sequen$\mathrm{ce}=1$.

22. Busta ER, Mancher M, Cuff PA, McAdam K, Keusch $\mathrm{G}$, editores. Integrating Clinical Research into Epidemic Response: The Ebola Experience. Washington, D.C.: National Academies Press; 2017.

23. Calain P. The Ebola clinical trials: a precedent for research ethics in disasters. J Med Ethics 2018; 44(1):3-8.

24. Baden LR, Rubin EJ, Morrissey S, Farrar JJ, Drazen JM. We Can Do Better - Improving Outcomes in the Midst of an Emergency. N Engl J Med 2017; 377:14821484.

25. Largent EA. EBOLA and FDA: reviewing the response to the 2014 outbreak, to find lessons for the future. $J$ Law Biosci 2016; 3(3):489-537. 
26. Dodd LE, Follmann D, Proschan M, Wang J, Malvy D, van Griensven J, Ciglenecki I, Horby PW, Ansumana R, Jiang J-F, Davey RT, Lane HC, Gouel-Cheron A. A meta-analysis of clinical studies conducted during the West Africa Ebola virus disease outbreak confirms the need for randomized control groups. Sci Transl Med 2019; 11(520):eaaw1049.

27. Falavigna M, Colpani V, Stein C, Azevedo LCP, Bagattini AM, Brito GV, Chatkin JM, Cimerman S, Dal Ben Corradi MF, Cunha CA, Medeiros FC, Oliveira Junior HA, Fritscher LG, Gazzana MB, Gräf DD, Marra LP, Matuoka JY, Nunes MS, Pachito DV, Pagano CGM, Parreira PCS, Riera R, Silva Júnior A, Tavares BM, Zavascki AP, Rosa RG, Dal-Pizzol F. Diretrizes para o tratamento farmacológico da COVID-19. Consenso da Associação de Medicina Intensiva Brasileira, da Sociedade Brasileira de Infectologia e da Sociedade Brasileira de Pneumologia e Tisiologia. Rev Bras Ter Intensiva 2020; 32(2):166-196.

28. Sanders JM, Monogue ML, Jodlowski TZ, Cutrell JB. Pharmacologic Treatments for Coronavirus Disease 2019 (COVID-19): A Review. JAMA 2020; 323(18):1824-1836.

29. International Coalition of Medicines Regulatory Authorities (ICMRA). Covid-19 [Internet]. 2020 [acessado 2020 set 20]. Disponível em: http://www.icmra. info/drupal/en/covid-19.

30. Rome BN, Avorn J. Drug Evaluation during the Covid-19 Pandemic. N Engl J Med 2020; 382:22822284.

31. International Coalition of Medicines Regulatory Authorities (ICMRA). ICMRA statement on clinical trials. 2020 [acessado 2020 set 20]. Disponível em: http://www.icmra.info/drupal/en/news/statement_ on_clinical_trials.

32. WHO Solidarity Trial Consortium. Repurposed Antiviral Drugs for Covid-19 - Interim WHO Solidarity Trial Results. N Engl J Med 2021; 384:497-511.

33. Pearson H. How COVID broke the evidence pipeline. Nature 2021; 593(7858):182-185.

34. Turner JR. New FDA Guidance on General Clinical Trial Conduct in the Era of COVID-19. Ther Innov Regul Sci 2020; 54:723-7724.

35. Food and Drug Administration (FDA). COVID-19 Real World Data (RWD) Data Elements Harmonization Project [Internet]. 2020 [acessado 2020 out 29]. Disponível em: https://www.fda.gov/drugs/coronavirus-covid-19-drugs/covid-19-real-world-data-rwddata-elements-harmonization-project.

36. European Medicines Agency (EMA). EMA's governance during COVID-19 pandemic [Internet]. 2020 [acessado 2020 set 8]. Disponível em: https://www. ema.europa.eu/en/human-regulatory/overview/public-health-threats/coronavirus-disease-covid-19/ emas-governance-during-covid-19-pandemic.

37. European Medicines Agency (EMA). Fast track procedures treatments and vaccines for COVID-19 [Internet]. 2020 [acessado 2020 out 20]. Disponível em: https://www.ema.europa.eu/en/documents/leaflet/ infographic-fast-track-procedures-treatments-vaccines-covid-19_en.pdf.
38. Hernandez AV, Roman YM, Pasupuleti V, Barboza JJ White CM. Hydroxychloroquine or Chloroquine for Treatment or Prophylaxis of COVID-19: A Living Systematic Review. Ann Intern Med 2020; 173(4):287296.

39. Mehra MR, Desai SS, Ruschitzka F, Patel AN. Retracted: Hydroxychloroquine or chloroquine with or without a macrolide for treatment of COVID-19: a multinational registry analysis. Lancet 2020; S01406736(20)31180-6.

40. Recovery Collaborative Group. Effect of Hydroxychloroquine in Hospitalized Patients with COVID-19: Preliminary results from a multi-centre, randomized, controlled trial. N Engl J Med 2020; 383(21):20302040.

41. Axfors C, Schmitt AM, Janiaud P, Van't Hooft J, Abd-Elsalam S, Abdo EF, Abella BS, Akram J, Amaravadi RK, Angus DC, Arabi YM, Azhar S, Baden LR, Baker AW, Belkhir L, Benfield T, Berrevoets MAH, Chen C-P, Chen T-C, Cheng S-H, Cheng C-Y, Chung W-S, Cohen YZ, Cowan LN, Dalgard O, Val FFAE, Lacerda MVG, Melo GC, Derde L, Dubee V, Elfakir A, Gordon AC, Hernandez-Cardenas CM, Hills T, Hoepelman AIM, Huang Y-W, Igau B, Jin R, Jurado-Camacho F, Khan KS, Kremsner PG, Kreuels B, Kuo C-Y, Le T, Lin Y-C, Lin W-P, Lin T-H, Lyngbakken MN, McArthur C, McVerry BJ, Meza-Meneses P, Monteiro WM, Morpeth SC, Mourad A, Mulligan MJ, Murthy S, Naggie S, Narayanasamy S, Nichol A, Novack LA, O'Brien SM, Okeke NL, Perez L, Perez-Padilla R, Perrin L, Remigio-Luna A, Rivera-Martinez NE, Rockhold FW, Rodriguez-Llamazares S, Rolfe R, Rosa R, Røsjø H, Sampaio VS, Seto TB, Shahzad M, Soliman S, Stout JE, Thirion-Romero I, Troxel AB, Tseng T-Y, Turner NA, Ulrich RJ, Walsh SR, Webb SA, Weehuizen JM, Velinova M, Wong H-L, Wrenn R, Zampieri FG, Zhong W, Moher D, Goodman SN, Ioannidis JPA, Hemkens LG. Mortality outcomes with hydroxychloroquine and chloroquine in COVID-19 from an international collaborative meta-analysis of randomized trials. Nat Commun 2021; 12(1):2349.

42. Food and Drug Administration (FDA). Coronavirus (COVID-19) Update: FDA Reiterates Importance of Close Patient Supervision for 'Off-Label' Use of Antimalarial Drugs to Mitigate Known Risks, Including Heart Rhythm Problems [Internet]. 2020 [acessado 2020 abr 29]. Disponível em: https://www.fda.gov/news -events/press-announcements/coronavirus-covid19-update-fda-reiterates-importance-close-patientsupervision-label-use?utm_campaign $=20200429 \% 20$ MCMi\&utm_medium=email\&utm_source=Eloqua.

43. Kupferschmidt K. Three big studies dim hopes that hydroxychloroquine can treat or prevent COVID-19 [Internet]. Science; 2020 [acessado 2020 jun 30]. Disponível em: https://www.sciencemag.org/news/2020/06/ three-big-studies-dim-hopes-hydroxychloroquinecan-treat-or-prevent-covid-19.

44. European Medicines Agency (EMA). COVID-19: chloroquine and hydroxychloroquine only to be used in clinical trials or emergency use programmes [Internet]. 2020 [acessado 2020 set 8]. Disponível em: https:// www.ema.europa.eu/en/news/covid-19-chloroquine -hydroxychloroquine-only-be-used-clinical-trials-emergency-use-programmes. 
45. European Medicines Agency (EMA). COVID-19: reminder of risk of serious side effects with chloroquine and hydroxychloroquine [Internet]. 2020 [acessado 2020 set 8]. Disponível em: https://www.ema.europa. eu/en/news/covid-19-reminder-risk-serious-side-effects-chloroquine-hydroxychloroquine.

46. Wang Y, Zhang D, Du G, Du R, Zhao J, Jin Y, Fu S, Gao L, Cheng Z, Lu Q, Hu Y, Luo G, Wang K, Lu Y, Li H, Wang S, Ruan S, Yang C, Mei C, Wang Y, Ding D, Wu F, Tang X, Ye X, Ye Y, Liu B, Yang J, Yin W, Wang A, Fan G, Zhou F, Liu Z, Gu X, Xu J, Shang L, Zhang Y, Cao L, Guo T, Wan Y, Qin H, Jiang Y, Jaki T, Hayden FG, Horby PW, Cao B, Wang C. Remdesivir in adults with severe COVID-19: a randomised, double-blind, placebo-controlled, multicentre trial. Lancet 2020; 395(10236):1569-1578.

47. Food and Drug Administration (FDA). Emergency Use Authorization [Internet]. 2021 [acessado 2021 maio 14]. Disponível em: https://www.fda.gov/emergency -preparedness-and-response/mcm-legal-regulatory -and-policy-framework/emergency-use-authorization\#coviddrugs.

48. European Medicines Agency (EMA). First COVID-19 treatment recommended for EU authorization [Internet]. 2020 [acessado 2020 set 8]. Disponível em: https://www.ema.europa.eu/en/news/first-covid19-treatment-recommended-eu-authorisation.

49. European Medicines Agency (EMA). Human Regulatory. Conditional Marketing Authorization [Internet]. 2020 [acessado 2020 set 8]. Disponível em: https:// www.ema.europa.eu/en/human-regulatory/marketing-authorisation/conditional-marketing-authorisation.

50. Brasil. Portaria GM no 188 , de 3 de fevereiro de 2020. Declara Emergência em Saúde Pública de importância Nacional (ESPIN) em decorrência da Infecção Humana pelo novo Coronavírus (2019-nCoV). Diário Oficial da União 2020; 04 fev.

51. Agência Nacional de Vigilância Sanitária (Anvisa). RDC no 351, de 20 março de 2020. Dispõe sobre a atualização do Anexo I da Portaria SVS/MS no 344 , de 12 de maio de 1998, e dá outras providências. Diário Oficial da União 2020; 20 mar.

52. Agência Nacional de Vigilância Sanitária (Anvisa). RDC no 405, de 22 de julho de 2020. Estabelece as medidas de controle para os medicamentos que contenham substâncias constantes do Anexo I desta Resolução, isoladas ou em associação, em virtude da Emergência de Saúde Pública de Importância Internacional (ESPII) relacionada ao novo Coronavírus (SARS-CoV-2). Diário Oficial da União 2020; 23 jul

53. Agência Nacional de Vigilância Sanitária (Anvisa). Nota técnica sobre cloroquina e hidroxicloroquina [Internet]. 2020 [acessado 2020 out 29]. Disponível em: https://www.gov.br/anvisa/pt-br/arquivos-noticias -anvisa/830json-file-1.

54. Agência Nacional de Vigilância Sanitária (Anvisa). Nota de esclarecimento sobre a ivermectina [Internet]. 2020 [acessado 2020 out 29]. Disponível em: https:// www.gov.br/anvisa/pt-br/assuntos/noticias-anvisa/2020/nota-de-esclarecimento-sobre-a-ivermectina.
55. Brasil. Ministério da Saúde (MS). Protocolo de manejo clínico da Covid-19 na Atenção Especializada. Brasília: MS; 2020.

56. Brasil. Ministério da Saúde (MS). Nota Informativa 5/2020 - DAF/SCTIE/MS.MS [Internet]. 2020 [acessado 2020 out 29]. Disponível em: https://www.saude. gov.br/images/pdf/2020/marco/30/MS---0014167392 ---Nota-Informativa.pdf.

57. Governo do Brasil. Brasil Imunizado. Rendesivir. Registro Condicional [Internet]. [acessado 2021 maio 12]. Disponível em: https://www.gov.br/pt-br/noticias/ saude-e-vigilancia-sanitaria/2021/03/anvisa-aprova -registro-de-segunda-vacina-contra-o-coronavirus.

58. Agência Nacional de Vigilância Sanitária (Anvisa). Voto $n^{\circ}$ 115/2021/DIRE5 [Internet]. [acessado 2021 maio 12]. Disponível em: https://www.gov.br/anvisa/pt-br/composicao/diretoria-colegiada/reunioesda-diretoria/votos/2021/copy_of_rextra-6-de-2021/ voto-115-2021-dire5.pdf.

59. Comissão Nacional de Incorporação de Tecnologias (CONITEC). Diretrizes Brasileiras para Tratamento Hospitalar do Paciente com COVID-19. Protocolos Clinicos e Diretrizes Terapêuticas. Relatório de Recomendação maio de 2021. CONITEC; 2021.

60. Thomson K, Nachlis H. Emergency Use Authorizations During the COVID-19 Pandemic: Lessons From Hydroxychloroquine for Vaccine Authorization and Approval. JAMA 2020; 324(13):1282-1283.

61. Goyal PK, Mathur R, Medhi B. Understanding the challenges and ethical aspects of compassionate use of drugs in emergency situations. Indian J Pharmacol 2020; 52(3):163-171.

62. Halimi V, Daci A, Stojanovska S, Panovska-Stavridis I, Stevanovic M, Filipce V, Grozdanova A. Current regulatory approaches for accessing potential COVID-19 therapies. J Pharm Policy Pract 2020; 13:16.

Artigo apresentado em 20/11/2020

Aprovado em 26/05/2021

Versão final apresentada em 28/05/2021

Editores-chefes: Romeu Gomes, Antônio Augusto Moura da Silva 